\title{
Clinical Usefulness of Cancer Antigen 15-3 in Breast Cancer Patients Before and After Surgery
}

\author{
Antonella Daniele ${ }^{*}, 1$, Rosa Divella ${ }^{1}$, Paolo Trerotoli ${ }^{2}$, Maria Elena Caringella ${ }^{1}$, Angelo Paradiso ${ }^{3}$, \\ Porzia Casamassima ${ }^{1}$, Ines Abbate ${ }^{1}$, Michele Quaranta ${ }^{1}$ and Antonio Mazzocca ${ }^{4}$ \\ ${ }^{I}$ Department of Experimental Oncology, Clinical Chemistry Laboratory, National Cancer Institute, I.R.C.C.S. "Giovanni \\ Paolo II", Bari, Italy \\ ${ }^{2}$ Department of Biomedical Science and Human Oncology, University of Bari, Medical School, Bari, Italy \\ ${ }^{3}$ Scientific Directorate of the National Cancer Institute, I.R.C.C.S. "Giovanni Paolo II", Bari, Italy \\ ${ }^{4}$ Department of Emergency and Organ Transplantation, Section of Internal Medicine, Allergology and Clinical \\ Immunology, University of Bari School of Medicine, Bari, Italy
}

\begin{abstract}
Background: Cancer Antigen 15-3 (CA 15-3) is a tumor-associated antigen used as serum marker for breast cancer surveillance in patients and for monitoring the response to treatment. Aim of this study was to retrospectively evaluate CA 15-3 as a prognostic factor in early detection of breast cancer relapse as well as to analyze the statistical correlation between CA 15-3 levels and clinical-pathological parameters including staging, grading, estrogen and progesterone receptors.

Methods: Sera of 726 women with breast carcinoma obtained preoperatively and postoperatively were assayed for CA 15 3 by chemoluminescent immunometric assay.

Results: We found that the mean serum CA 15-3 levels in patients before surgery were significantly higher $(36.59 \mathrm{U} / \mathrm{ml})$ compared with those of CA 15-3 after surgery $(27.11 \mathrm{U} / \mathrm{ml})$. We also found that elevated preoperative serum levels of CA 15-3 were significantly correlated with the presence of metastatic disease. In particular, among 305/700 patients (43,6\%) that displayed over cut-off ( $>40 \mathrm{U} / \mathrm{ml})$ preoperative levels of CA 15-3, 94 patients $(30,8 \%)$ developed advanced disease (metastases to distant sites). By contrast, in a subgroup of 395/700 patients (56.4\%) with CA 15.3 serum levels $<40 \mathrm{U} / \mathrm{ml}$, only 32/305 patients (8\%) showed signs of advanced disease during follow-up. Cox regression analysis revealed that only the presence of metastasis and the increased serum levels of CA 15-3 after surgery are significant risk factors for relapse of disease.

Conclusion: Elevated preoperative concentrations of CA 15-3 may be a useful predictive factor for cancer progression in postoperative patients.
\end{abstract}

Keywords: CA 15-3, prognostic marker, follow-up, metastasis, breast cancer.

\section{INTRODUCTION}

CA $15-3$ is a mucinous carbohydrate antigen product of the MUC1 gene, originally identified by two monoclonal antibodies: DF3 raised against a membrane fraction of breast liver metastases and 115D8 raised against milk fat globule membrane $[1,2]$. The antigenic component is an epitope of Polymorphic Epithelial Mucin (PEM), a high molecular weight $(300-400 \mathrm{kDa})$ heavily glycosylated protein encoded by the MUC1 gene [3]. The polymorphism is associated with protein components that are expressed in repeated sequences that can vary between 20 and 75 times. Recent data suggest that MUC1 plays a role in cell adhesion (leading to decreased cell-cell and cell-extracellular matrix interactions)

*Address correspondence to this author at the Department of Experimental Oncology, National Cancer Institute "Giovanni Paolo II", Viale Orazio Flacco, 6570124 Bari, Italy; Tel: +39-80-5555321; Fax: +39-80-5555259; E-mail: Antonella27@inwind.it immunity and metastasis $[4,5]$. Increased expression levels of MUC1 in primary tumor suggest that this protein acts as anti-adhesive molecule and facilitates detachment of malignant cells, both from adjacent cells and extracellular matrix (ECM) in primary cancer [6]. Thus, MUC1 may play a role in the initiation of cancer invasion and metastatic dissemination. In fact, recent evidence shows that breast cancer growth and dissemination is impaired in MUC1 deficient mice [7]. MUC1 blocks tumor cells escaping immuno-surveillance as demonstrated by several studies showing that soluble MUC1 inhibits the killing of MUC1expressing cells by cytotoxic T lymphocytes (CTL). High levels of circulating MUC1 found in some patients with breast cancer may be involved in blocking tumor cells escaping immune-surveillance. Moreover, cancer cells expressing high levels of MUC1 may be less vulnerable to immune attack since the cell membrane-associated MUC1 inhibits the immune recognition process of CTL and lymphokine activating killer (LAK) cells by masking 
antigens involved in this process on cell surface $[4,5]$. CA 15-3 is currently the most widely used serum marker for breast cancer [8]. In particular, it is recommended in the evaluation of response to therapy and in monitoring the breast cancer outcome.

So far, there is no evidence for efficacy of screening with this marker in breast cancer. CA $15-3$ is elevated in only $3 \%$ of patients with localized cancer whereas it is elevated in up to $70 \%$ of patients with metastatic disease [8]. CA 15-3 is not a suitable marker in screening high-risk population for the presence of cancer, however, it may be useful as a prognostic marker in breast cancer patients.

The elevation of CA $15-3$ (values over $120 \mathrm{kU} / \mathrm{L}$ ) is found in over $30 \%$ of breast cancer patients with advanced disease [3]. Serum CA 15-3 has been extensively studied mainly to monitor the response of breast cancer to the treatment $[9,10]$ or to detect early relapse in breast cancer follow-up. In fact, CA 15-3 serum levels were found higher in about $70 \%$ of patients with progressive disease, whereas they were decreased in $80 \%$ of patients with regression of disease [11]. Few studies have been currently published on the ability of CA 15-3 to predict the survivability in breast cancer patients. Duffy and co-workers reported that patients with increased preoperative concentrations of CA 15-3 had a worse prognosis than those with low concentrations [12]. The aims of this retrospective study are: 1) to evaluate the usefulness of tumor marker CA 15-3, determined at the time of primary diagnosis, as prognostic factor in breast cancer; 2) to compare CA 15-3 with other clinical parameters, such as age, diagnosis, ER and $\mathrm{PgR}$ status, stage of disease, tumor size $(\mathrm{T})$, lymph node status $(\mathrm{N})$, the presence of metastases (M) and administered therapy.

\section{PATIENTS AND METHODS}

\section{Patients}

We enrolled 726 consecutive patients with breast carcinoma and analyzed serum samples obtained from patients who underwent radical mastectomy or conservative surgery (quadrantectomy + axillary dissection) at Department of the Tumor Institute "Giovanni Paolo II" of Bari between January 1996 and May 2004. All breast cancer patients were staged according to TNM - UICC classification [13]. Information concerning age, menopausal status, familiarity, diagnosis, type of surgery, therapy administered, and clinical pathology such as tumor size (T), lymph node status $(\mathrm{N})$, the presence of metastases $(\mathrm{M})$ and estrogen (ER) or progesterone receptor $(\mathrm{PgR})$ status, for each patient were collected through clinical charts. (Table 1). Treatments administered to breast cancer patients is summarized in Table 2. CA15-3 serum levels were determined by IMMULITE 2000 BR-MA (Medical System), a two steps sequential chemoluminescent immunometric assay that used an anti-CA15-3 mouse monoclonal antibody. The lower limit of sensitivity was $0.2 \mathrm{U} / \mathrm{ml}$ and the established cut-off was $40 \mathrm{U} / \mathrm{ml}$. According to cut-off, patients were divided in two subgroups: a) subgroup one consisting of 395/700 patients (56.4\%) with CA $15-3$ serum levels $\leq 40 \mathrm{U} / \mathrm{ml}$ and b) subgroup two consisting of $305 / 700$ patients (43.6\%) with CA $15-3>40 \mathrm{U} / \mathrm{ml}$. Sensitivity (percentage) of CA 15-3 correlated to stages of disease is shown in Table $\mathbf{3}$.
Table 1. Main Clinical-Pathological Characteristics of $\mathbf{7 2 6}$ Breast Cancer Patients Selected from 2,163 Cases

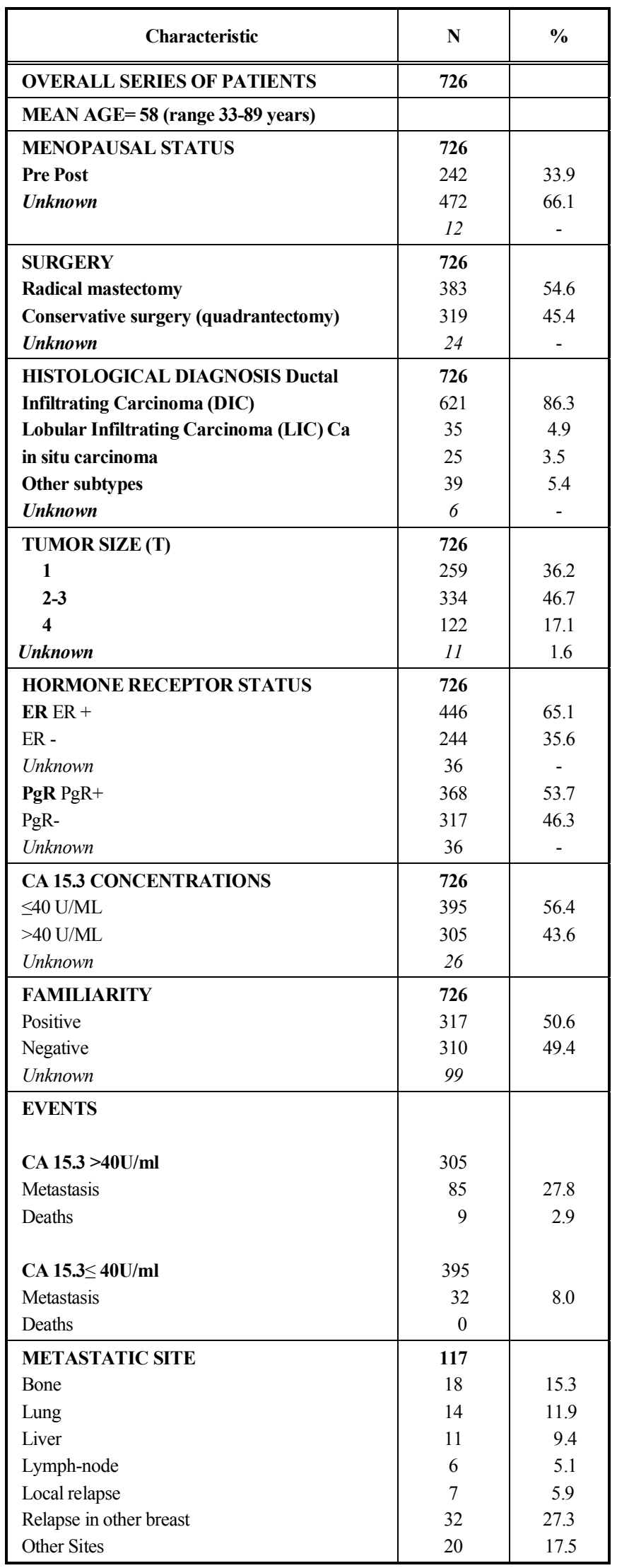


Table 2. List of Treatments Administered to Breast Cancer Patients Enrolled for the Study

\begin{tabular}{|l|c|c|}
\hline \multicolumn{1}{|c|}{ Treatment } & N & \% \\
\hline \hline No Therapy & 156 & 21.4 \\
\hline Hormonal therapy alone & 56 & 7.7 \\
\hline Radiotherapy alone & 15 & 2.0 \\
\hline Chemotherapy alone & 14 & 1.9 \\
\hline Hormonal + chemotherapy & 108 & 14.8 \\
\hline Hormonal + radiotherapy & 18 & 2.5 \\
\hline Chemotherapy + radiotherapy & 40 & 5.5 \\
\hline Hormonal + chemotherapy +radiotherapy & 310 & 42.6 \\
\hline Herceptin + chemotherapy & 7 & 1.0 \\
\hline Unknown Total & 2 & 0.2 \\
\hline
\end{tabular}

Table 3. Sensitivity of CA $\mathbf{1 5 . 3}$ are Correlated to Stage of Disease. Sensitivity is Expressed as Percentage of Subjects Diagnosed as Having Cancer Among Patients with Cancer

\begin{tabular}{|c|c|}
\hline Stage & Sensitivity (\%) \\
\hline \hline I & $5-15$ \\
\hline II & $15-25$ \\
\hline III & $25-35$ \\
\hline IV & $75-95$ \\
\hline
\end{tabular}

\section{Immunohistochemistry (IHC) for Hormone Receptors}

Immunohistochemistry for ER and $\mathrm{PgR}$ was performed using primary monoclonal antibodies: anti-human ER 1D5 (DAKO) and anti-human PgR 1A6 (YLEM). Patients with intraductal infiltrating carcinoma (IDC) with known reactivity for the antibodies were used as positive control. Sections of 4-6 $\mu \mathrm{m}$ obtained from paraffin embedded tissues were placed onto the slide, pre-treated with endogenous peroxidase and blocked with hydrogen peroxidase.

Sections were subjected to an antigen retrieval procedure using microwave irradiation at 650 Watt for 3 cycles for 8 min and citrate buffer ( $10 \mathrm{mM}$ Sodium Citrate, $0.05 \%$ Tween 20, $\mathrm{pH}$ 6.1). The slides were then incubated with streptavidin-biotinylated AB (StreptABComplex) overnight at $4{ }^{\circ} \mathrm{C}$ and the reaction was developed with Ammino-EthylCarbazolo (AEC). The results were recorded as the percentage of positively stained target cells. Samples with $>10 \%$ positively stained tumor cells were considered positive.

\section{STATISTICAL ANALYSIS}

The relation between qualitative variables was assessed by $\chi$ test; McNemar test was used to compare percentage of paired data and the agreement was measured with index $k$ of
Cohen. The quantitative variables were summarized as mean and $95 \%$ Confidence Interval (CI) if Gaussian distributed or using median and Interquatile range (IQR) if not Gaussian distributed. Correlation between quantitative variable Gaussian distributed was performed by means of Pearson correlation coefficient. Survival was estimated by KaplanMeyer curves [14]. Statistically significant differences in survival between the two subgroups (patients with CA15-3 serum levels $\leq 40 \mathrm{U} / \mathrm{ml}$ and patients with CA15-3 serum levels $\geq 40 \mathrm{U} / \mathrm{ml}$ ) were assessed by the log-rank test. Cox model was performed to assess the risk of death or other event related to the presence of prognostic features [15]. Age was entered to the model as continuous variable to adjust hazard ratio. Analysis were carried out using SAS (V8.2) software (SAS Institute Inc., Cary, NC, USA).

\section{RESULTS}

Our analysis focused on 726 patients with breast carcinoma. The mean age was 58 years (range 33-89 years) and $621 / 720$ patients $(86.3 \%)$ were diagnosed histologically as ductal infiltrating carcinoma. Tumor size was classified as T1 (Tumor size less than or equal to 2 centimeters) in 259/715 (36.2\%), T2-T3 (Tumor size between 2 and 5 centimeters) in 334/715 (46,7\%) and T4 (Tumor extends to chest wall) in $122 / 715(17.1 \%)$ of cases according to the TNM classification. The percentage of patients with negative lymph nodes was $47.1 \%$ (337/716) whereas the percentage of patients with positive nodes was $48.5 \%$ (347/716). The median follow-up time was 25 months (range 0-98 months). The progression of disease was observed in 117/616 patients $(19.0 \%)$ whereas only $9 / 616$ deaths $(1.46 \%)$ were reported. Main characteristics of 726patients enrolled in the present study are shown in Table $\mathbf{1}$.

CA 15-3 serum levels were evaluated preoperatively and postoperatively (at 3 monthly intervals for the first 5 years, at 6 monthly intervals for the next 2 years and yearly with regular intervals) in each patients. Mean serum value of CA $15-3$ prior to surgery was significantly higher $36.59 \mathrm{U} / \mathrm{ml}(\mathrm{CI}$ 95\% 34.5-38.9) as compared to mean value of CA 15-3 after surgery $27.11 \mathrm{U} / \mathrm{ml}$ (CI 95\% 24.3-28.8). 305/700 of patients (43.5\%) had higher serum levels of CA 15-3 before treatment (consisting in hormonal, radio-, chemo- or other therapy) whereas only $83 / 405$ of cases (20.49\%) displayed values over cut-off after treatment. We found that decreased serum levels of CA 15-3 in these patients were statistically significant (Test McNEmar. 67.7; $\mathrm{p}<0.0001$ ). In addition, we found a significant direct correlation between CA 15-3 serum levels before and after surgery $\left(\chi^{2}=0.62 p<0.0001\right)$. No statistically significant difference in Disease Free Survival (DFS) was found in univariate analysis by means of log-rank test for the following factors: 1) categories of CA $15-3$; 2) type of therapy administered; 3) ER and $\mathrm{PgR}$ receptor; 4) familiarity; 5) type of surgery (quadrantectomy vs mastectomy); 6) histological diagnosis; 7) menopausal status and 8) HER-2/neu expression.

In DFS, we found a statistically significant difference between patients classified as T1 compared to T2-T3 (logrank: $5.74 ; p=0.016)$ as well as between lymph nodenegative $(\mathrm{N}-)$ and lymph node-positive $(\mathrm{N}+)$ patients $(\mathrm{log}-$ rank: 4.55; $\mathrm{p}=0.033)$. The difference in survival between 
patients with absence (M0) and presence of metastatic spread (M1) was also statistically significant (log rank: 67.5; $\mathrm{p}<0.0001)$. We also found that, before surgery, a subgroup of patients $(94 / 305 ; 30.8 \%)$ with CA $15-3$ values over cut-off showed progression of disease or death compared with the subgroup of patients $(32 / 395 ; 8 \%)$ with CA $15-3$ levels less than $40 \mathrm{U} / \mathrm{ml}(\chi=14.9 ; \mathrm{p}<0.0001)$. As shown in Fig. (1), Kaplan Meier curves show a statistically significant difference in survival for breast cancer patients with CA 153 values over cut-off and for patients with normal values of CA 15-3 (log-rank $=7.48 ; \mathrm{p}=0.0062$ ). The Kaplan Meier overall survival in a subgroup of patients with levels of CA 15-3 over cut-off was of 67 months compared to patients with normal CA 15-3 levels showing a longer survival (log rank: $=34.39 ; \mathrm{p}<0.0001)$. The number of metastases or death events were higher in patients $(94 / 305 ; 30.8 \%)$ with over cut-off CA 15-3 levels compared with those (32/395;
$8 \%)$ with CA $15-3$ less than $40 \mathrm{U} / \mathrm{ml} \times(\chi=53.56, \mathrm{p}<$ 0.0001). Multivariate Cox model showed that independent variables (among Tumor, Nodes, Metastasis, ER and PgR status, familiarity, menopausal status considered) were the presence of metastasis, CA 15-3 values after surgery and menopausal status. The multivariate model was statistically significant (score $=43.41 ; \mathrm{p}<0.0001$ ) and the risk of disease relapse or death was 5.3 for high levels of CA 15-3after surgery (CI 95\% 1.88-14.89), 5.19 for the presence of metastasis (CI 95\% 1.02-26.41) and 4.83 for menopausal status (CI95\% 1.18-19.67). An interaction factor was included in the model to adjust the hazard ratio due to the correlation between the presence of metastasis and CA15-3 levels (Table 4). The risk of disease relapse in the presence of metastatic cancer was 33.9 (CI95\% 7.3-158.1) and for CA $15-3$ over cut-off was 6.6 (CI 95\% 2.8-15.4).

\section{Ca $15.3 \leq 40 U / m I$}

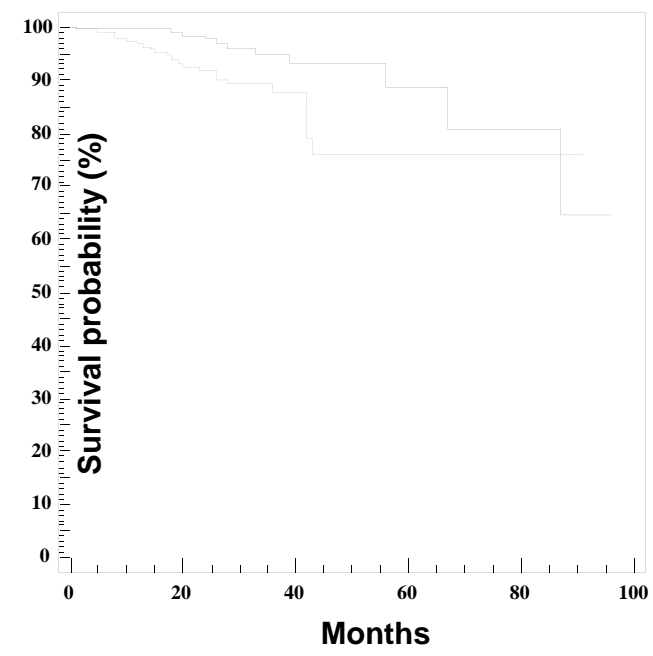

\section{Ca $15.3>40 U / m I$}

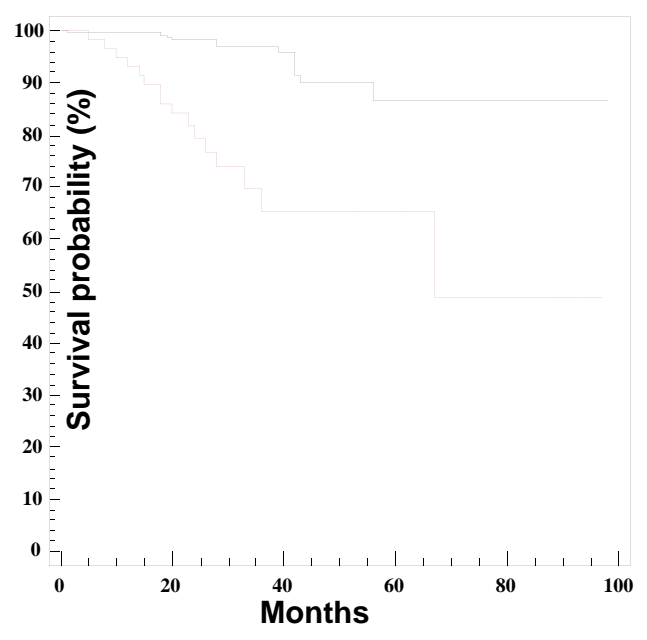

Fig. (1). Kaplan Meier Curves for Disease Free Survival (DFS): death, metastasis, relapse.

Table 4. Hazard Ratio of Univariate and Multivariate Cox Model After Stepwise Selection

\begin{tabular}{|c|c|c|c|c|c|c|c|}
\hline \multirow{2}{*}{ Risk Factors } & \multirow{2}{*}{} & \multicolumn{2}{|c|}{ Univariate Analysis } & \multicolumn{3}{|c|}{ Multivariate Analysis } \\
\cline { 3 - 7 } & & HR & CI 95\% & p & HR & CI 95\% & p \\
\hline \hline & DIC $v s$ others & 0,65 & $0,29-1,42$ & 0,28 & 0,44 & $0.09-2.16$ & 0.31 \\
\hline LIC $v s$ others & 1,76 & $0,54-5,77$ & 0,35 & 0,85 & $0.12-5.98$ & 0.87 \\
\hline Histological diagnosis & In situ $v s$ others & 1,45 & $0,35-6,04$ & 0,61 & 0,83 & $0.07-9.85$ & 0.88 \\
\hline Stage T & T1 $v$ s others & 0,34 & $0,14-0,82$ & 0,02 & 0,59 & $0.17-2.06$ & 0.42 \\
\hline Stage N & N0 $v s$ others & 0,37 & $0,17-0,78$ & 0,009 & 0.78 & $0.29-2.14$ & 0.64 \\
\hline Stage M & M1 $v s$ others & 15,85 & $6,5-38,66$ & $<0,0001$ & 5.19 & $1.02-26.41$ & 0.04 \\
\hline Estrogen Receptor & Present $v s$ absent & 1,32 & $0,61-2,85$ & 0,48 & 1.26 & $0.47-3.36$ & 0.64 \\
\hline Progesterone Receptor & Present $v s$ absente & 0,88 & $0,44-1,78$ & 0,72 & 0.61 & $0.23-1.62$ & 0.32 \\
\hline Familiarity & Present $v s$ absente & 0,76 & $0,39-1,52$ & 0,44 & 1.01 & $0.41-2.46$ & 0.99 \\
\hline Menopausal status & Present $v s$ absente & 1,56 & $0,71-3,43$ & 0,26 & 4.83 & $1.18-19.67$ & 0.03 \\
\hline Ca 15.3 before surgery & $>=40$ UI $v s<40$ UI & 2,61 & $1,28-5,32$ & 0,008 & 1.28 & $0.45-3.69$ & 0.64 \\
\hline Ca 15.3 after surgery & $>=40$ UI $v s<40$ UI & 6,92 & $3,27-14,66$ & $<0,0001$ & 5.3 & $1.88-14.89$ & 0.001 \\
\hline
\end{tabular}




\section{DISCUSSION}

Breast cancer is the most prevalent carcinoma among women in Western Countries. In Italy, $10 \%$ of all women are diagnosed with breast cancer during their lifetime. About 33,000 women result currently estimated to be affected by breast cancer, 11,000 women die from breast cancer each year, and 30,000 survive after 5 years since primary diagnosis [16, 17]. Breast cancer mortality can only be reduced by detecting the tumor at the earlier stage. For example, self-palpation breast examination, a screening procedure used in an attempt to detect early breast cancer, has proved to be effective in discovering $80 \%$ of all carcinomas treated surgically [18]. Serum markers in breast cancer are helpful for clinicians in providing more effective management of the disease. To this end, different markers have been proposed in the last years. In particular, carcinoembryonic antigen (CEA) and MUC1 (CA 15-3) are the most widely used and investigated in the breast cancer follow-up. Initial studies indicate that CA 15-3 is abnormal in the majority of patients with metastatic breast cancer and that antigen levels are correlated with changes in the clinical status of breast cancer patients $[19,20]$. However, CA 15-3 is not recommended as screening tool in early detection for breast cancer, even though it remains an important asset to monitor the efficacy of medical therapies after surgery. We observed that the sensitivity of CA 15-3 increases to $95 \%$ only in patients with advanced disease. In fact, according to the current literature, CA $15-3$ is not specific or sensitive enough to detect early breast cancer. Elevation of CA15-3 is not a reliable index in the diagnosis of breast cancer since normal serum levels can also be found in women with breast cancer. However, CA15-3 seems to be a reliable prognostic predictor for monitoring disease progression. As in our study, Colomer and co-workers showed that circulating levels of CA 15-3 can be elevated in patients with cancer and that serial determinations of CA 15-3 may be useful in the post-surgical follow-up of breast cancer patients when specific types of benign disease that may cause increase of this antigen are excluded [21].

The main use of CA $15-3$ is to monitor the breast cancer patient response to treatment and for early breast cancer recurrence or metastasis. CA 15-3 can be used as a marker only if cancer is producing elevate amounts of it. However, it may be useful as a prognostic marker even in a small percentage of patients with localized breast cancer showing increased levels of CA 15-3. If CA 15-3 is initially elevated may be used to monitor treatment and when repeated on a regular basis, to detect early recurrence; CA 15-3 is not useful when breast cancer is detected early by others examinations. In general, higher levels of CA 15-3 are correlated with a larger tumor burden and a more advanced disease. The serum levels of CA 15-3 increase as cancer develops. In metastatic breast cancer, the highest levels of CA 15-3 are often seen when the cancer has spread to the bones and /or to the liver. In fact, Wojtacki and co-workers demonstrated that increased levels of CA 15-3in patients without clinical evidence of breast cancer recurrence are a strong indicator of the presence of occult metastatic disease. Therefore, an accurate screening for metastatic disease should be the procedure of choice in breast cancer patients with NED (Not Evidence of Disease) and elevated CA 15-3 levels [22]. Accordingly, an initial elevation of CA 15-3 that does not return to the normal range, is an indicator of lack of response to treatment and represents an adverse prognostic factor. Our study show a significant correlation between CA 15-3 and the response to treatment. In fact, we demonstrated a continuous increase of the marker in patients with metastatic disease that had subsequently developed locoregional relapse by analyzing CA 15-3 serum concentrations in breast cancer patients during follow-up. Similar observations were reported by different authors [2327]. For example, Geraghty and coll. reported that patients with increased levels of CA 15-3 show a significantly shorter latency in developing metastatic disease compared with normal tumor marker levels $(20.8+/-3.3$ versus $10.3+/-2.7$ months, respectively; $\mathrm{P}<0.03$ ). Moreover, CA $15-3$ values at diagnosis were increased in $88 \%$ of 115 patients with metastatic disease [28].

In line with our findings, Horobin and co-workers showed that high preoperative levels of CA 15-3 can predict adverse outcome in patients with stage I and stage II breast cancer. In particular, these authors reported that the median value of CA $15-3(43 \mathrm{kU} / 1)$ in 26 patients with stage IV disease $(86 \%$ patients with bone metastasis) was significantly higher than the median for 97 patients classified as stage I or II (17 kU/I) [28]. Our study showed that patients presenting CA15-3 levels over 40U/ml (cut-off) have both worse disease free interval (DFS) and overall survival (OS) compared to those with concentrations $<40 \mathrm{U} / \mathrm{ml}$. This is supported by Iaffaioli and co-workers that reported that patients with high preoperative levels of CA 15-3 (>30 $\mathrm{U} / \mathrm{ml}$ ) have shorter disease-free interval than patients with lower levels [28]. The present study also demonstrated that preoperative CA 15-3 levels are significantly associated with early recurrences of the disease, thus adding useful information to prognosis especially in lymph node-positive patients. CA 15-3 can also provide prognostic information in patients with advanced breast cancer. For example, Berutti and co-workers showed that patients with CA 15-3 value $<30 \mathrm{U} / \mathrm{ml}$, at the time of the first recurrence, survived significantly longer than those with concentrations of CA 153 higher than the aforementioned value [29]. The aim of this retrospective study was to determine the applicability of serum CA 15-3 assay in the detection of breast cancer at the time of primary diagnosis and during the follow up. Our results demonstrated that CA 15-3 levels are frequently higher before surgery than after excision of primary tumor. In addition, we found that higher levels of CA 15-3 in patients prior to surgery are more frequently associated with disease progression and worse outcome compared to patients with lower levels of CA 15-3. The most likely explanation can be that patients with elevated levels of CA 15-3 may harbor micrometastatic disease undetectable with the standard diagnostic procedures. Using multivariate and univariate analysis, we show that the only independent variables are increased levels of CA 15-3 and the presence of metastases. Finally, our study demonstrated the important role of this marker in monitoring the efficacy of post-surgery therapies (i.e. chemo, hormonal or radiotherapy).

In conclusion, the present study suggests that higher levels of CA 15-3 would be a reliable prognostic marker for women affected by breast carcinoma. Moreover, CA 15-3 
could be a useful marker for monitoring disease activity in those patients undergoing aggressive treatments.

\section{CONFLICT OF INTEREST}

The authors confirm that this article content has no conflict of interest.

\section{ACKNOWLEDGEMENTS}

Declared None.

\section{REFERENCES}

[1] Crommelin DJA, Schellkens H. From clone to clinic. Kluwer Academic Publishers, 11122, 1990.

[2] Schmidt-Rhode P, Schulz KD, Sturm G, Raab-frick A, Prinz H. CA 15-3 in breast cancer: first experience with a new monoclonal test system. Med Sci Res 1987; 15: 7656.

[3] Duffy MJ. CA 15-3 and related mucins as circulating markers in breast cancer. Ann Clin Bio Chem 1999; 36 (Pt-5): 579-86.

[4] Hilkens J, Ligtenberg M, Vos Hl, Litinou SV. Cell membraneassociated mucins and their adhesion modulating properties. Trends Biol Sci 1992; 17: 359-63.

[5] Taylor-Papadimitriou J, Burchell J, Miles DW, Dalziel M. MUC1 and cancer. Biochim Biophys acta 1999; 1455: 301-13.

[6] Wessling J, vander Valk SW, Hilkens J. A mechanism for inhibition of E-cadherin-mediated cell-cell adhesion by the membrane-associated mucin episialin/MUC1. Mol Biol Cell 1996; 7(4): 565-77.

[7] Spicer AP, Rowse GJ, Linder TK, et al. Delayed mammary tumor progression in MUC1 null mice. J Biol Chem 1995; 270: 30093101.

[8] Duffy MJ. Biochemical markers in breast cancer: which ones are clinically useful. Clin Biochem 2001; 34: 347-52

[9] Tumor Marker Expert panel. Clinical practice guidelines for the use of tumor markers in breast and colorectal cancer. J Clin Oncol 1996; 14: 2843-77.

[10] Stearns V, Yamauchi H, Haydes DH. Circulating tumor markers in breast cncer: accepted utilities and novel prospects. Breast Cancer Res Treat 1998; 52: 239-59.

[11] Bast RC, Ravdin P, Hayes DF, et al. 2000 update of recommendations for the use of tumor markers in breast and colorectal cancer: clinical practice guidelines of the American Society of Clinical Oncology. J Clin Onc 2001; 19(6): 1865-78.

[12] Duffy MJ, Shering S, Sherry F, Mc- Dermott E, O' Higging N. CA 15-3: a prognostic marker in breast cancer. Int J Biol Mark 2000; $15: 330-3$.

[13] Hermanek P, Sobin LH, Eds. International Union Against Cancer (UICC): TNM classification of malignant tumors, $4^{\text {th }}$ ed. Berlin: Springer Verlag 1987.
[14] Kaplan EL, Meyer P. Nonparametric estimation from incomplete observations. J Am stat Assoc 1958; 53: 457-81.

[15] Cox DR. Statistical significance tests. Br J Clin Pharm 1982; 14: 325-31.

[16] Micheli A, Mugno E, Krogh V, et al. Cancer prevalence in European Registry areas. Ann Oncol 2002; 13(6): 840-65.

[17] Verdecchia A, Mariotto A, Capocaccia R, et al. Incidence and prelevante of all cancerous diseases in Italy trends and implications. Eur J Cancer 2001; 37(9): 1149-57.

[18] Moskowitz M, Gartside PS. Evidence of breast cancer mortality reduction: aggressive screening in women under age 50. AJR 1982; 138: 911-6.

[19] Hayes DF, Zurawsky WF, Kufe DW. Comparison of circulating CA 15-3 and carcinoembryonic antigen in patients with breast cancer. J Clin Oncol 1986; 4: 1541-50.

[20] Todini C, Hayes DF, Gelman R, et al. Comparison of CA 15-3 and carcinoembryonic antigen in monitoring the clinical course of patients with metastatic breast cancer. Cancer Res 1988; 48: 410712.

[21] Colomer R, Ruibal A, Salvador L. Circulating tumor marker levels in advanced breast carcinoma correlate with the extent of metastatic disease. Cancer 1989; 64(8): 1674-81.

[22] Wojtachi J, Dziewulska-Bokiniec A. Serum CA 15-3 determinations in the postoperative follow-up of breast cancer patients. Libri Oncol 1995; 24(3): 147-52.

[23] Bombardieri E, Pizzichetta M, Verollesi P, et al. CA 15-3 determination in patients with breast cancer: clinical utility for the detection of distant metastases. Eur J Cancer 1993; 29: 144-6.

[24] Geraghty JG, Coveney EC, Sherry B, O’ Higgins NJ, Duffy MJ. CA 15-3 in patients with locoregional and metastatic breast carcinoma. Cancer 1992; 70: 2831-8.

[25] Kerin MJ, Mc Anena OJ, O’ Malley VP, Grimes H, Given HF. CA 15-3: its relationship to clinical stage and progression to metastatic disease in breast cancer. Br J Surg 1989; 76: 838-9.

[26] Miserez AR, Günes I, Müller-Brand J, Walther E, Fridrich R, Mäcke $H$. Clinical value of a mucin-like carcinoma associated antigen in monitoring breast cancer patients in comparison with CA 15-3. Eur J Cancer 1991; 27: 126-31.

[27] Horobin JM, Browning MCK, Mc Forlane NP, et al. Potential use of tumor marker CA 15-3 in the staging and prognosis of patients with breast cancer. J R Coll Edinb 1991; 36: 219-21.

[28] Iaffaioli RV, Caponigro F, Esposito G, et al. Impact of preoperative CA 15-3 levels in operable breast cancer. Comparison with tissue polypeptide antigen (TPA) and carcinoembryonic antigen (CEA). Int J Biol Markers 1991; 6: 21-4.

[29] Berruti A, Tampellini M, Torta M, Buniva T, Gorzegno G, Dogliotti L. Prognostic value in predicting overall survival of two mucinous markers: CA 15-3 and CA 125 in breast cancer patients at first relapse of disease. Eur J Cancer 1994; 30A: 2082-4.

Received: November 21, 2011

Revised: December 30, 2011

Accepted: January 9, 2012

(C) Daniele et al.; Licensee Bentham Open.

This is an open access article licensed under the terms of the Creative Commons Attribution Non-Commercial License (http://creativecommons.org/licenses/by-nc/ 3.0/) which permits unrestricted, non-commercial use, distribution and reproduction in any medium, provided the work is properly cited. 